\title{
LA TERAPIA COMUNITARIA INTEGRATIVA COMO HERRAMIENTA EFICAZ EN SITUACIONES DE DESASTRES NATURALES
}

\section{THE INTEGRATIVE COMMUNITY THERAPY AS AN EFFECTIVE TOOL IN SITUATIONS OF NATURAL DISASTERS}

MARÍA ALEXANDRA VALAREZO LOAYZA ${ }^{1}$

Recibido: 15 de mayo de 2017

Aceptado: 29 de agosto de 2017

\footnotetext{
${ }^{1}$ Pontificia Universidad Católica del Ecuador, Facultad de Psicología, Quito, Ecuador (mvalarezo652@puce.edu.ec).
} 



\section{LA TERAPIA COMUNITARIA INTEGRATIVA COMO HERRAMIENTA EFICAZ EN SITUACIONES DE DESASTRES NATURALES.}

\section{THE INTEGRATIVE COMMUNITY THERAPY AS AN EFFECTIVE TOOL IN SITUATIONS OF NATURAL DISASTERS}

María Alexandra Valarezo Loayza

Términos: Terapia Comunitaria Integrativa, resiliencia, Rueda Comunitaria, terremoto

Terms: Integrative Community Therapy, resiliency, Community Rounds, earthquake

\section{RESUMEN}

El terremoto del 16 de abril de 2016 que afectó al litoral ecuatoriano, demandó de asistencia psicológica inmediata para sus pobladores. Fueron las Ruedas de Terapia Comunitaria Integrativa, herramientas de trabajo exitosas, que permitieron, in situ inicialmente y en lo posterior en albergues, comunidades y escuelas, abrir un espacio para que los pobladores pudieran reunirse a hablar de sus sufrimientos y preocupaciones, logros y celebraciones. Participaron los damnificados, profesionales, voluntarios, brigadistas, de instituciones públicas y privadas. En un compartir horizontal y circular se favoreció el empoderamiento de la comunidad, autoafirmados como sujetos activos poseedores de saberes propios, recuperaron su autoestima personal y comunitaria. Dejaron de ser víctimas, para ser sujetos activos, corresponsables y partícipes de las soluciones. 
Se establecieron actitudes resilientes y estrategias de superación personal y co- lectiva, se crearon redes solidarias de participación y organización.

\section{ABSTRACT}

The earthquake of april 16 (2016) that affected the Ecuadorian coast, demanded immediate psychological assistance for its inhabitants. It was the Integrative Community Therapy rounds, tools of successful work, that allowed, initially at the site and later in shelters, communities, and schools, the opening of a space so that the inhabitants could meet to talk about their sufferings and concerns as well as their achievements and celebrations. In the wheels participated victims, professionals, volunteers, emergency teams, those belonging to public and private institutions. In a horizontal and circular sharing session was favored community empowerment, self-affirmed as active subjects and possessors of their own abilities and knowledge, they were able to recover their personal and community self-esteem. This model took them out of victimization to become active subjects, co-responsible, and participants in the solutions. It was established resilient attitudes and strategies for personal and collective improvement, favoring the creation of solidary networks of participation and organization.

\section{INTRODUCCIÓN}

Afrontar la demanda urgente de asistencia psicológica a las personas afectadas por el terremoto del 16 de abril de 2016 en la provincia de Manabí y Esmeraldas, motivó a una decisión inmediata de viajar y atender a quienes estaban refugiados de forma emergente en el albergue de Portoviejo.

La realidad psicosocial de esos primeros momentos daba cuenta de altos niveles de confusión, desorganización, angustia, miedo, incertidumbre de lo que vendrá, terror a que se repita el terremoto, inseguridad por estar viviendo a la intemperie, con el peligro que los ladrones se lleven lo poco que han recibido, pues perdieron todo lo que tenían. Su vida estaba transformada, albergados en carpas improvisadas, con gente desconocida. Debieran aceptar reglas im- 
puestas por extraños que rotaban continuamente, con grandes ofrecimientos que difícilmente se cumplían.

Félix Cova (2010) habla sobre los terremotos y tsunami de Chile, muestra similares situaciones a las vividas en Ecuador. La población enfrentada a la inseguridad social, carencia de servicios, desorganización, dolor y muerte. Aumentan en los pobladores los estresores pues su vida se ha trastocado por la destrucción, la incertidumbre, frustración frente a la indolencia o el aprovechamiento político. Bolton \& Hill, (1996) expresan que la comunidad pierde la vivencia del mundo como un lugar seguro y predecible, pierden la confianza en los demás, en sí mismos y en el futuro.

Es la Terapia Comunitaria Integrativa Sistémica (TCl) la que permitió un acogimiento inmediato, in situ, a los diversos grupos humanos que demandaban asistencia psicológica: familias damnificadas, líderes comunitarios espontáneos, profesionales de diversas ramas, voluntarios de todo el país, que colaboraron con la incipiente organización del albergue.

La TCl es una metodología de trabajo creada en Brasil en 1986 por los doctores Adalberto Barreto, médico psiquiatra y su hermano Airton Barreto', abogado.
En su libro "La Terapia Comunitaria Integrativa paso a paso" (2013) el Dr. Adalberto cuenta sus experiencias en el Proyecto 4 Varas, Pirambú, Fortaleza. Los hermanos Barreto atendían individualmente a una innumerable población, víctimas de abandono y miseria humana. Ambos viéndose sobrepasados por la cantidad de gente que demandaba su ayuda profesional en el Proyecto 4 Varas, optaron por reunir a todos en una rueda en la que pudieran hablar de sus dolencias y dificultades. A partir de esta nueva modalidad, deciden fundar el Movimiento Integral de Salud Mental Comunitaria y consecuentemente la TCl.

Esta escucha comunitaria dio cuenta que las soluciones estaban en la misma gente, en su sabiduría popular. Barreto (2008) define a la TCl como "una práctica que posibilita la creación de redes solidarias, movilizando recursos y competencias de las personas, familias y comunidades, suscitando la dimensión terapéutica del grupo"

Su eficacia se basa en una amplia comprensión de la realidad social de la comunidad, entendiéndola como un "tejido común en el cual las personas se reconocen a sí mismas y al otro, como un ser semejante, en un lugar de pertenencia y de construcción de identidad. En un

\footnotetext{
${ }^{1}$ Barreto Adalberto de Paula, Dr. en Psiquiatría y Antropología, Lcdo. en Filosofía y Teología, Francia. Profesor de Salud Comunitaria en la Universidad Federal de Ceará, Brasil. Abarret1@matrix.com.br. Barreto Airton, Dr. en Abogacía
} 
interjuego entre acción y emoción se van organizando las formas de pensar, sentir y actuar de la comunidad." (Barreto, 2008)

La TCl fue extendiendo su campo de acción a una diversidad de grupos vulnerables: pobreza, enfermedad, migración, discriminación social, ampliándose luego a grupos de profesionales, organizaciones sociales, comunitarias, gubernamentales, entre otros. Actualmente la $\mathrm{TCl}$ es una política pública en Brasil que se ha extendido a 27 estados brasileños, y se han formado alrededor de 36500 terapeutas comunitarios. Además, se ha extendido por varios países de Latinoamérica, Europa y África.

A Ecuador llegó en el año 2012, con la visita a nuestro país de Terapeutas Comunitarios del MISMEC DF, Movimiento Integrado de Salud Comunitaria del Distrito Federal. En ese año se hicieron las primeras ruedas comunitarias en Quito y Puyo. El trabajo realizado con los Abuelitos de la Calle, conjuntamente con practicantes de Psicología Clínica de la PUCE fue eficaz. Se demostró que los abuelos redujeron significativamente sus niveles de depresión (Bermúdez, 2014). En el año 2014 se funda el Centro de Formación de Terapia Comunitaria MUYUMPA y se imparte la primera for- mación en Quito. Actualmente hay alrededor de 60 Terapeutas Comunitarios formados en Ecuador.

Las ruedas comunitarias de la $\mathrm{TCl}$ son el espacio en donde se acoge a la comunidad para hablar sobre sus dificultades y sus logros, valorar sus experiencias de vida, rescatar su identidad y ampliar su percepción de las dificultades y posibilidades, mediante una red de apoyo. Tiene una duración aproximada de $1 \frac{1}{2}$ hs y no hay límite en el número de participantes. Se han realizado ruedas hasta de 450 personas. Es una rueda abierta en la que pueden entrar o salir quienes participan en ella.

Tanto en Ecuador como en países que han enfrentado la traumática vivencia de un terremoto, la realidad y la forma de vida se transforman en un caos. Ante ello el Dr. Adalberto Barreto plantea la Rueda Comunitaria como:

"Un momento de transformación, transmutación del caos ${ }^{2}$ para experimentar el kairós ${ }^{3}$, el espacio sagrado donde cada uno reorganiza su discurso y resignifica su sufrimiento dando origen a una nueva lectura de los elementos que lo provocan. En esta dimensión sagrada de transfor-

\footnotetext{
${ }^{2}$ Caos: de raíces griegas y latinas, se define como lo impredecible, desorden, confusión. (DRAE, 2017)

${ }^{3}$ Kairos: término del lenguaje griego que significa "momento adecuado u oportuno" Hesíodo lo definió como "todo lo que es mejor que algo" y Eurípides lo definió como "el mejor guía en cualquier actividad humana" (DRAE, 2017)
} 
mar el sufrimiento en crecimiento, la carencia en capacidad, la TCl adquiere la relevancia de un espacio sagrado"

El término Terapia viene del griego Terapeia que significa acoger, acompañar. Comunitaria, se define como un conjunto de personas que tienen algo en común, viven juntos, tienen los mismos intereses o viven bajo las mismas reglas. El Dr. Adalberto Barreto plantea lo comunitario como la común unión de quienes conviven bajo un mismo modo de vida. Integrativo, cuya definición latina dice "Hacer un todo o conjunto con partes diversas". En la TCl se integran los saberes de los diversos contextos socioculturales de los participantes, saberes personales $y$ ancestrales que se ponen en juego durante la rueda y se integran en un saber comunitario, que fortalece su identidad y los empodera como miembros activos de su comunidad.

Sistémica en tanto se aborda el sistema social como una compleja red de relaciones humanas interconectadas e interdependientes, en la que cada sujeto influye en los demás, es parte activa de un gran todo llamado comunidad, caracterizada por ser multicultural.

La situación de un terremoto destruye en cada individuo su esquema de vida y de identidad. Al perder todo lo material y gran parte de sus víncu- los, pierde los referentes que le dan un sentido de identidad, de pertenencia a un grupo humano, a un espacio físico, a un modo de vida. A nivel de asistencia social, pone a prueba la capacidad de organización y coordinación de sistemas de ayuda, que en su gran mayoría, no se hallan preparados para la atención emergente de un desastre natural, provocando un caos de instituciones que pretenden cumplir con su función. Mc Farlane y Novis (2006) plantean como desastre "un evento potencialmente traumático que es colectivamente experimentado, tiene un inicio agudo y una delimitación temporal. Raquel Cohen (2000) plantea que los damnificados son aquellas personas afectadas por un desastre o un evento natural inesperado y altamente devastador. Este afecta de manera indiscriminada a toda la población, sin diferenciación de edad, sexo, nivel socioeconómico ni raza. Las repercusiones en las víctimas dan lugar a una crisis, que afecta a nivel físico, emocional, mental, familiar, social y espiritual.

Frente a esta realidad, la TCl convocó a todos los afectados: niños, adolescentes, adultos, personas de la tercera edad, profesionales, voluntarios, entre otros, a sentarse juntos a conversar sobre su realidad de dolor y sufrimiento, así como de sus saberes y habilidades. Ofrecimos un espacio de organización y reflexión en el cual se recupera la autoes- 
tima individual y grupal, la capacidad de resiliencia, el empoderamiento, a pesar del dolor y las múltiples pérdidas materiales y humanas sufridas. Reafirmando el planteamiento del Dr. Barreto, las capacidades y soluciones están en la misma gente, en su sabiduría popular.

La TCl se basa en 5 pilares teóricos: el pensamiento sistémico, la pedagogía de Paolo Freire, la resiliencia, la antropología cultural y la teoría de la comunicación.

El primer pilar es el pensamiento sistémico (Costa, 2012) afirma que "las crisis y los problemas apenas pueden ser entendidos y presentar una solución, si los percibimos como pedazos integrados de una red compleja, la cual crea ramificaciones uniendo y relacionando a las personas en un todo que envuelve a lo biológico (cuerpo), psicológico (mente y emociones) y la sociedad". (Bertalanfly, et al 1976). Somos un todo en el que cada parte es interdependiente de las demás. Dice Barreto que para enfrentar la vida debemos ser conscientes de esta interrelación entre lo personal, familiar, social y comunitario. Bertalanfly (1976) plantea que un elemento esencial del sistema es el amor. "Amor significa abrir un espacio para la existencia de una interacción mutua, solo así se puede alcanzar una verdadera socialización". Es la base de la socialización humana y cuando este falta, la sociedad termina descomponiéndose como tal. Es impresionante como el amor, el amor humano, unido al amor divino (cualquiera sea la creencia), el amor a la vida, a su gente, a su tierra, a pesar de que ha temblado y destruido lo que se había construido en ella, es lo que mueve y transforma, tanto a los damnificados, como a quienes fueron a apoyarlos.

El segundo pilar de la $\mathrm{TCl}$ es la Teoría de la Comunicación, Según Idalberto Chiavenato (2006) comunicación es "el intercambio de información entre personas. Significa volver común un mensaje o una información. Constituye uno de los procesos fundamentales de la experiencia humana y la organización social"En la TCl la comunicación verbal o no verbal, permite el intercambio de experiencias y emociones, con un lenguaje común, que permite integrar la diversidad y la posibilidad de dar un significado y sentido a las vivencias humanas que son comunes a todos.

El tercer pilar es la Antropología Cultural (Barreto, 2008) plantea la importancia de la cultura, a partir de la cual cada miembro de un grupo basa su habilidad para pensar, discernir y establecer. Resalta los valores culturales y las creencias como factores importantes en la formación de la identidad y del grupo (Barreto, 2008). En la TCl se pone de manifiesto el acervo cultural, sus raíces y costumbres. Como ejemplo el cantar 
el pasillo Manabí en la rueda, fortaleció su identidad grupal y recuperó sus sentimientos de orgullo manaba.

El cuarto pilar es la Pedagogía de Paulo Freire, quien comprende el proceso de enseñanza aprendizaje como acción / reflexión / acción, cada uno enseña y aprende al mismo tiempo. Aporta un cambio en la forma de ver su realidad a cada participante, respetando las diferencias, incluyendo los diversos saberes, fortaleciendo la credibilidad y la esperanza en la posibilidad de construir un mundo igualitario y más justo (Barreto, 2010). En la TCl se comparten historias de vida de manera horizontal, respetando el dolor y compartiendo los modos de resolver las dificultades. Todos poseen saberes adquiridos a partir de sus sufrimientos, convertidos en habilidades y capacidades. Andrew Palva (2004), respecto del pensamiento de Paulo Freire y la educación liberadora, postula que educar implica leer no solo la palabra sino también leer el mundo, con un pensamiento crítico, con el objetivo de actuar como sujetos de cambio. En la TCl, cada participante es un sujeto activo y no una víctima pasiva de su realidad.

Finalmente la resiliencia, entendida por H. Camarotti (2014) como la capacidad de transformar el trauma en crecimiento, el sufrimiento en capacidades, es decir, reconstruirse positivamente a partir del enfrentamiento de las adver- sidades. La resiliencia familiar es percibida como la posibilidad de la familia de alcanzar, después de una vivencia traumática, los aspectos positivos que preservan la credibilidad de cada uno y de todos los miembros de la familia (Delac y Cyrulnik, citado por Cammarotti). Las ruedas de TCI facilitan a sus participantes recuperar su capacidad de resiliencia. Las soluciones están en ellos, no en quienes los apoyan. Se busca validar el saber popular, inserto en cada grupo humano

Cada encuentro en la rueda de $\mathrm{TCl}$ se constituyó en un acto terapéutico en sí, por su poder transformador. Esta metodología de trabajo es eficaz en situaciones críticas. Permite actuar in situ, no requiere de condiciones físicas determinadas, es viable en lugares inusuales como albergues, iglesias, espacios públicos, plazas o en sitios en los que la comunidad frecuenta o vive.

La TCl también permite trabajar con todo tipo de población tanto en edad como en clase social, cultural, académica, etc. Su versatilidad permite el trabajo en todos los niveles de necesidad. En el caso del terremoto, permitió trabajar con los damnificados, con los grupos de voluntarios, familias, profesionales de todas las áreas, docentes, en los primeros días y meses después del terremoto. Permite un acompañamiento multinivel, y acoge todas las necesidades de la comunidad. 


\section{METODOLOGÍA}

La aplicación de la TCl en la zona del desastre se dio a los pocos días del terremoto del 16 de abril. Las ruedas de $\mathrm{TCl}$ en un inicio, a los 6 días del terremoto, fueron ideales para el trabajo en grupo. En la situación devastadora en la que estaban viviendo, apareció la posibilidad de sentarse juntos a hablar de sus preocupaciones y sufrimientos, así como de sus logros y celebraciones. A pesar del gran dolor que vivían la alegría permanecía en ellos, la gratitud por la vida que Dios les había otorgado, que a otros les había quitado. Su inmensa nobleza y solidaridad les hacía estar preocupados por todas aquellas personas que no tenían un albergue, por los niños que perdieron a sus padres. Una profunda fe en Dios los mantenía con la esperanza de que iban a salir adelante.

Este trabajo da cuenta de 17 ruedas de TCl realizadas por 6 Terapeutas Comunitarios voluntarios desde abril 2016 hasta enero 2017 en Quito, Portoviejo, Canoas, El Carmen, Charapotó, Pedernales y Las Lagunas.

La metodología de la rueda de $\mathrm{TCl}$ se caracteriza por la simplicidad de su estructura. Se trabaja con un Terapeuta Comunitario y 1 o 2 coterapeutas y consta de 6 etapas:

1. Acogida: se inicia con un ritual de bienvenida que permite potenciar el sentido de pertenencia, relajar y expresar emociones. Se explica qué "es un espacio donde se reúne la comunidad para hablar de sus problemas, dificultades, así como de sus logros y realizaciones. La comunidad tiene problemas pero también tiene alternativas de solución." (Barreto, 2010). Luego se invita a celebrar todo aquello que la comunidad desee: cumpleaños, aniversarios, la vida, logros, etc.

En las primeras ruedas realizadas en el albergue de Portoviejo, a los pocos días del terremoto, los participantes proponían festejar la oportunidad que les ha dado Dios de estar vivos y estar juntos. La vida fue valorada como lo más preciado.

El siguiente paso es el planteamiento de las reglas:

a. Silencio activo.

b. Hablar en primera persona usando el pronombre Yo.

c. Los AJIS: no aconsejar, no juzgar, no interpretar, no sermonear

d. Se solicitan aportes culturales: canciones, versos, proverbios, chistes, dichos pertinentes al tema que se está tratando.

e. Respetar la historia de vida de cada persona. 
Dinámica de integración: se pide a los participantes aportar con una actividad corta que ayude a la integración. Los aportes fueron múltiples: juegos tradicionales, dinámicas grupales entre otros.

2. Elección del Tema: el terapeuta pide a los participantes hablar sobre aquello que les preocupa y les trae sufrimiento (la piedra en el zapato que no los deja estar bien). Sea respecto de lo personal, familiar, laboral, social, comunitario, etc. Una vez que se han planteado varios temas, se abre un espacio de identificación con lo propuesto y se procede a la votación. Se invita a quien presentó el tema elegido a hablar ampliamente sobre su preocupación.

3. Contextualización: el grupo hace preguntas para ampliar la comprensión de la problemática.

4. Problematización: se abre la palabra para el grupo, planteando "quién ya vivió una situación así y qué hizo para resolverla"Todos pueden participar y aportar con sus soluciones y posibilidades de enfrentar la situación.

En este paso los participantes comparten sus historias de vida, las per- las obtenidas a partir de los traumas o crisis vitales. Se crea un ambiente de respeto, en el que no son juzgados ni aconsejados, sino validados en sus aprendizajes y capacidades.

5. Rituales de integración y connotación positiva: se invita al grupo a hacer una rueda en la que todos en un abrazo comparten la vivencia de estar sostenidos, balancearse sin caer, porque está el grupo o la comunidad que los sostiene. El Terapeuta recoge los aportes de quienes compartieron sus vivencias y connota positivamente. Los participantes verbalizan sus aprendizajes alcanzados durante la rueda. Es un momento especial, emocional y espiritualmente, las personas rescatan los valores, las creencias, su fe en sí mismos, en el grupo y en el futuro.

Doña Santa dice "estoy agradecida porque hoy es un nuevo día para resucitar de nuevo, para cantar, reír, para volver a ser feliz"

6. Evaluación: el equipo de Terapeuta y coterapeutas se reúne a dar su apreciación de lo vivido y el registro de los logros y dificultades, planteándose cómo lo harían mejor. 


\section{PARTICIPANTES}

El trabajo realizado a partir del 23 de abril con la TCl, estuvo dirigido a:

- Rueda 1: personas damnificadas de las carpas 1 y 2 en el albergue de Portoviejo,

- Rueda 2: coordinadores de carpas en el albergue de Portoviejo

- Rueda 3: psicólogos, parvularios y voluntarios en el albergue de Portoviejo

- Rueda 4,5: personas damnificadas que fueron trasladadas a albergues en Quito

- Rueda 6: personas damnificadas en Pedernales

- Rueda 7: docentes de la escuela Alajuela, en el Pueblito de Charapotó

- Rueda 8 y 9: profesionales de todas las ramas en Quito, que deseaban ir a Manabí

- Rueda 10: madres de familia de la escuela Alajuela, Pueblito de Charapotó
- Rueda 11: albergados en El Carmen, Iglesia Evangélica Metodista Unida del Ecuador. Como parte del EMAH de Chile

- Rueda 12: voluntarios que estuvieron en la zona., Invitados por la Cruz Roja Ecuatoriana, Quito

- Rueda 13: albergados en escuela de El Carmen. Personal del MIES.

- Rueda 14: albergados y personas que asistían a un programa social, invitados por MetropolitanTouring

- Rueda 15: familia compuesta por 5 miembros, padre, madre, 2 adolescentes varones y una niña de 10 años. Charapotó

- Rueda 16: docentes en la escuela de Las Lagunas, Charapotó

- Rueda 17: padres de familia de la escuela y comunidad de Las Lagunas, Charapotó 


\section{POBLACIÓN ATENDIDA}

Tabla 1. Participantes de las ruedas de TCl en Manabí, terremoto del 2016.

\begin{tabular}{|c|c|c|c|c|c|}
\hline RUEDA & PARTICIPANTES & HOMBRES & MUJERES & CIUDAD & LUGAR \\
\hline 1 & 20 & 5 & 15 & PORTOVIEJO & ALBERGUE \\
\hline 2 & 15 & 3 & 12 & PORTOVIEJO & ALBERGUE \\
\hline 3 & 14 & 1 & 13 & PORTOVIEJO & ALBERGUE \\
\hline 4 & 10 & 6 & 4 & QUITO & ALBERGUE \\
\hline 5 & 9 & 5 & 4 & QUITO & ALBERGUE \\
\hline 6 & 33 & 5 & 28 & PEDERNALES & ALBERGUE \\
\hline 7 & 14 & 4 & 10 & CHARAPOTÓ & ESCUELA \\
\hline 8 & 10 & 2 & 8 & QUITO & CONSULT \\
\hline 9 & 32 & & 32 & CHARAPOTÓ & ESCUELA \\
\hline 10 & 9 & 2 & 7 & QUITO & CONSULT \\
\hline 11 & 45 & 12 & 33 & EL CARMEN & IGLESIA \\
\hline 12 & 37 & 9 & 28 & QUITO & CRUZ ROJA \\
\hline 13 & 86 & 19 & 67 & EL CARMEN & MIES \\
\hline 14 & 90 & 26 & 64 & EL CARMEN & MIES \\
\hline 15 & 5 & 3 & 2 & CHARAPOTÒ & CASA \\
\hline 16 & 14 & 3 & 11 & LAS LAGUNAS & ESCUELA \\
\hline 17 & 40 & 19 & 21 & LAS LAGUNAS & TINGLADO \\
\hline TOTAL & 483 & 119 & 364 & & \\
\hline
\end{tabular}

Fuente: elaboración propia

Se ha trabajado con 483 personas está compuesta en su mayoría por jóveen 17 ruedas de Terapia Comunitaria, nes y adultos y en menor cantidad percon una proporción de 3 a 1 de mujeres sonas de la tercera edad y niños. respecto de los hombres. La población 


\section{MEDICIÓN}

Los temas que fueron expuestos para trabajar en las 17 ruedas de TCl están referidos a miedo, angustia, inseguridad, ansiedad, desesperación, tristeza, decepción, dolor del corazón, temor a que se repita el terremoto, a las réplicas, al tsunami. Su preocupación es por los fallecidos, los desaparecidos, por la familia, por lo material, por quienes no están recibiendo ayuda, por la depresión de la gente y su inconformidad, por la enfermedad física y mental, por el futuro, por los recuerdos de lo vivido, por los niños que se han quedado huérfanos. Por mi gente, mis vecinos, mi ciudad destruida, por la falta de trabajo, por la droga entre los jóvenes.

Los aprendizajes o perlas ${ }^{4}$ logradas se refieren a los siguientes aspectos:

a. Empoderamiento y fortalecimiento personal: "estoy viva, no puedo estancarme, he aprendido a ser fuerte y reanimarme", "ver el lado positivo de lo que toca vivir", "tenemos a la familia y a la comunidad más unida que antes" "Mis hijos son mi valor, mis padres son mi apoyo, seguimos por los que hemos quedado vivos" "Debemos dejar el miedo y luego, ya nada nos detendrá" "Debo encontrar mi centro, eso me fortalece, ahora sé de lo que soy capaz" "Hay que pisar en piedras firmes y honestas, para no caer en falso" "No hace falta que tenga respuestas para todas las necesidades que se presenten" "Estoy llena de las cosas que recibí del grupo" "Hay que vivir un día por vez, hoy vivamos alegres junto a los que quedamos" "Me di cuenta que antes ya superé problemas más difíciles, entonces creo que puedo superar este" "El miedo solo se vence enfrentándolo"

b. Apoyo social: "ayuda mucho contarle a alguien de confianza mis miedos y preocupaciones", "hay que llegar a las personas que se ven mal y hablar con ellas"'"los compañeros me dan fuerza y valor para seguir, solo unidos lo lograremos" "me motiva hacer las cosas por los demás, porque Jesús es verbo y no sustantivo"' $\mathrm{He}$ comprendido que no se puede ser el pilar de todos, yo también necesito ayuda" "Debo buscar información oficial y no hacer caso de los rumores"

c. Apoyo espiritual: "mi fuerza motivadora es Dios, me sostengo de la mano de Él", "gracias a Dios estamos

\footnotetext{
${ }^{4} \mathrm{En}$ la TCl se usa la metáfora de las perlas. Cuando un grano de arena ingresa en una ostra, es una amenaza para ella, para defenderse tiene que producir el nácar con el que recubre al grano de arena. A mayor dolor, mayor cantidad de nácar, más grande la perla. La perla es una herida cicatrizada. Así los dolores sufridos se convierten en habilidades y capacidades. (Barreto, 2015)
} 
vivos, otros ya no" "pido a Dios fuerzas para seguir adelante, por mis hijos y mi familia que queda viva" "hoy es un nuevo día, hemos resucitado, volvimos a nacer gracias a mi Dios, por eso hay que cantar, reír y volver a ser feliz" "tengo confianza en que Dios nos va a acompañar en este proceso de recuperación"

\section{RESULTADOS}

Aplicar la $\mathrm{TCl}$ en los momentos más críticos de un terremoto facilitó la creación de redes solidarias de sostén. Las soluciones no están en los terapeutas, están en la misma comunidad, ellos no son víctimas, sino sujetos activos, recreadores de su realidad. El aprendizaje es horizontal y mutuo. Todos aprendemos de la sabiduría de todos. Este modelo genera autonomía, pues son los damnificados los que se han empoderado de sus recursos personales, comunitarios, culturales y se van a apoyar en su propia autoestima individual y grupal. Los terapeutas facilitan este encuentro.

Varios ejemplos se pueden ver en los pobladores. Doña Carmen, albergada por varios meses en Portoviejo, costurera, lo perdió todo. A pesar de ello, con ayuda de la comunidad, recibió una máquina de coser, empezó a coser para los albergados, arreglándoles la ropa donada, ajustando la talla, cobraba centavos, porque ahí la gente no tenía dinero ni trabajo. Armó un taller de costura en el albergue, más adelante enseñó a las jóvenes del albergue, en un taller de pro- ducción de ropa. Ahora, fuera del albergue continúa trabajando y enseñando, favoreciendo a la comunidad con sus saberes.

La familia de Marco, joven de 16 años, se encontraba paralizada, un mes después del terremoto con el terror de las réplicas y de que su casa se termine de caer, sin recursos para sobrevivir ya que sus padres se quedaron sin trabajo, los lugares que los empleaban desaparecieron, la angustia los ha enfermado. En la rueda con la familia, Marco, el segundo hijo de 4, planteó la idea de vender papas fritas y hamburguesas en su barrio. Nadie vende eso en su zona. Con esta idea, la familia se organizó. Con ayuda de los voluntarios ahora tienen su carrito de comida en la plaza de El Pueblito de Charapotó. Toda la familia trabaja ahí, los padres en las horas en que los hijos van al colegio. Los jóvenes después de hacer sus tareas y en feriados. La hija menor reparte los pedidos a domicilio en su bicicleta. Ahora llevan su negocio a las ferias y fiestas de la zona. Confirmando lo que plantean Delae y CyrulniK, la resi- 
liencia familiar les permitió rescatar sus aspectos positivos, preservar la credibilidad en cada uno de sus miembros y en la familia como equipo.

Las madres de familia de la Escuela Alajuela, que en la rueda plantearon que sus maridos no tenían trabajo, se daban cuenta que no podían quedarse en la casa solo cuidando a los hijos, debían trabajar en lo que ellas saben hacer: vender menestras, empanadas, dulces. Al mes siguiente estaban participando del taller de telares con materiales reciclados, mediante formas de expresarse a través de la creación de sus propias artesanías.

En la ciudad de Quito se encontraba mucha gente deseosa de ir a prestar su ayuda a Manabí. Un grupo de profesionales y voluntarios solicitaron reunirse para hablar del terremoto. Se propuso realizar una rueda de TCI. El tema planteado fue el miedo de no saber con qué se iban a encontrar, sintiéndose poco preparados para asistir a los damnificados. Después de la rueda de TCI varios de ellos pudieron concretar su deseo y viajar a distintos puntos del país, llevando sus donaciones y aportando con sus saberes, entre ellos, la artista que llevó su taller de telares con material de reciclaje.

A continuación transcribo el testimonio de Camola, Artista Plástica, Terapeuta del Arte, voluntaria:
Asistí a la TCl a raíz del terremoto de abril 2016. Como todos con el deseo de ir a ayudar a la Costa, pero sin saber por dónde comenzar. Tenía un miedo paralizante, y a la vez, un sentimiento de obligación de prestar mi ayuda y culpa por no poder lograrlo. A pesar de haber ayudado en Tailandia luego del Tsunami de diciembre de 2004, ahora en Quito, no encontraba la fuerza para ir a ayudar a mi propia gente. Durante la rueda, el temor fue disminuyendo, me di cuenta que lo que cada persona pueda hacer es un aliciente, un apoyo y una pequeña sanación para las heridas de los damnificados. A la semana siguiente estuvimos con un grupo de voluntarios visitando la población costera de Charapotó. Como resultado de la $\mathrm{TCl}$ con mujeres, nació de ellas la necesidad de generar recursos para ayudar a sus familias. Propuse trabajar con ellas en varios talleres, enseñándoles a tejer el telar con materiales reciclados y naturales, ayudando a recobrar la creatividad y la confianza en ellas mismas. Al mismo tiempo recobrando mi fuerza y la certeza de la capacidad de cada ser humano. La TCI nos ayudó eficazmente a todos. 


\section{CONSIDERACIONES FINALES}

La fuente de conocimiento es la propia experiencia. Las carencias generan en las personas, habilidades y capacidades que les permiten avanzar. Afirma A. Barreto (2013) que "cualquier persona, cualquiera sea su condición socioeconómica y cultural, aunque lo ignore, posee saberes útiles para los demás." Revalorizamos el saber popular, el saber de la comunidad que se basa en sus vivencias y en sus conocimientos personales, ancestrales y culturales. Se propone valorizar este conocimiento personal, popular y complementarlo con el saber científico y académico.

Se ha podido constatar que la $\mathrm{TCl}$ es exitosa ya que facilita el procesamiento de las primeras etapas de la crisis de una manera funcional (Campana, E., Souza E., 2017), favorece la recuperación de su autoestima, fomenta la resiliencia, empodera a los participantes en sus capacidades y habilidades, en la identificación con su cultura y sus saberes ancestrales. Ahora se los apoya para que luego continúen por sí mismos su proceso de reconstrucción. En las ruedas cantamos al final, "me balanceo pero no me caigo, no me caigo porque usted está ahí." Es el grupo, la comunidad que identificada en un mismo dolor, en este caso ser damnificados del terremoto, se convierte en el sostén de cada uno de sus miembros.
La comunidad arma una red en la que todos se apoyan. Todos a un mismo nivel, tanto participantes, terapeutas, amas de casa, profesores, voluntarios, niños y abuelos. Entre todos se crean modelos de solución compartidos, la comunidad crea una nueva manera de ver las cosas y de caminar juntos. La rueda es eficaz porque tiene la magia de siempre funcionar, en sí misma es una terapia y cada uno se convierte en terapeuta de sí mismo.

"Yo no puedo darle nada que no exista ya dentro de usted. Yo no puedo presentarle ninguna otra galería de cuadros que la de su alma. No puedo dar a usted nada: solo la ocasión, el impulso, la clave. Yo he de ayudar a hacer visible su propio mundo; esto es todo". (Herman Hesse, 1927)

A nivel personal, el mayor aprendizaje que pude realizar a partir de estas experiencias de vida, ha sido el compartir con las personas afectadas por el terremoto, pues son maestros de vida, su fuerza inquebrantable me ha dado el testimonio de que toda dificultad puede ser superada. El hecho de tener vida es el mayor valor. Perder todo lo material permite encontrarse con lo esencial. La fortaleza espiritual es un pilar fundamental que da sentido a todo aquello que nos 
toca vivir. La realización personal en la tarea terapéutica está presente al abrirse a compartir el dolor, y en ese encuentro profundo, descubrir en las miradas, un nuevo sentido a lo que se siente y se piensa, una mirada que restituye el ser en lo más profundo, revitaliza el por qué seguir adelante y continuar en el camino de la vida.

Se dice que el dolor compartido es medio dolor, la alegría compartida es doble alegría. Poner en palabras aquello que enferma, ayuda a sanar el corazón y el cuerpo. "Cuando la boca calla, el cuerpo enferma. Dejemos que la boca hable para que el cuerpo y el corazón sanen", es el lema de la TCl
Haber podido expresar estas vivencias, me ayuda a sanar mi cuerpo, mi mente y mi corazón. Y a invitarles a que ustedes también pongan en palabras sus miedos y aquello que les preocupa y no los deja actuar. Y una vez reconocidos los miedos, puedan entregarse a la tarea psicoterapéutica sin temor, compartir de manera voluntaria su tiempo, pues cada vez hay más personas en situación de vulnerabilidad que necesitan de nuestro trabajo.

"La perla más hermosa encontrada después del terremoto del 16 de abril es el amor a la vida"

... y la tarea continúa. 


\section{BIBLIOGRAFÎA}

Barreto, A. P. (2013). Terapia Comunitaria Integrativa paso a paso. Fortaleza. Gráfica LCR.

Barreto A., Camarotti M.H., Freire T. (2013). A Terapia Comunitária Integrativa no Cuidado da Saúde Mental. Brasilia: Editora Krion.

Bermúdez, E. (2014) Apoyo emocional a las personas de la tercera edad desde la Terapia Comunitaria Integrativa. (Tesis de grado) Quito: PUCE.

Bertalanffy, L. V.(1976). Teoría general de los sistemas. México, DF, Fondo de cultura económica. Recuperado de http://biblioteca.udgvirtual. udg.mx/eureka/pudgvirtual/Teoriageneraldelossitemas_v4.pdf

Bolton \& Hill (1996), citado por Cova, F. Rincon, P. (2010), El terremoto y el tsunami del 27F y sus efectos en la Salud Mental. Terapia Psicológica. Vol 10. No. 2. pp 179-185. Recuperado de http://dx.doi.org/10.4067/s071848082010000200006

Camarotti, M. H. (2014). Resiliencia: El Poder de la Autotransformación. De la neurociencia a la evolución humana. Brasilia: Editora Kiron.

Camarotti, M. H, Guedes de Paula Freire T.C., Barreto, A. Org. (2011) Terapia Comunitaria Integrativa Sem
Fronteiras. Comprendendo suas interfaces e aplica OPS, Ed. Manual Moderno,ões. Brasilia. MISMEC-DF.

Camarotti, M.H. (2015). Conciencia autocurativa. Como utilizar seus mecanismos psicocerebrais para alcancar a cura. Brasilia. Editora Kiron.

Campana, E., Souza, E. (2017), Ruedas Vinculantes. Una nueva tecnología al servicio de la gente, Quito.

Chiavenato, I. (2006). Introducción a la teoría general de la administración. 7a.edición. Madrid: McGrawhill

Cohen, R. (2000). Salud mental para víctimas de desastres. Manual para trabajadores. México: OPS. Ed. El Manual Moderno.

Costa, E. (2012), Terapia Comunitaria Integrativa, Tesina, Brasilia: Brasil

Cyrulnik B. (2002) Los Patitos Feos. La Resiliencia: Una infancia infeliz no determina la vida. Barcelona. Editorial Gedisa.

Dias, M.D., Lazarte, R., Oliveira, F.F. (2013) Terapia Comunitária Integrativa, uma construção coletiva do conhecimento. Joao Pessoa. Ed. Universitaria

Gorovit, M. (2006). Desastres y trauma psicológico. Pensamiento Psico- 
lógico, vol 2, n. ${ }^{\circ}$, pp 15-39.

Hesse, H. (1927). El lobo estepario. Madrid: Alianza Editorial

Lazarte, R. (2011). Terapia Comunitaria. Reflexiones. João Pessoa. Ed. Universitaria

Mc Farlane, Novis (2006), citado por Cova, F., Rincón, P. (2010), El terremoto y el tsunami del $27 \mathrm{~F}$ y sus efectos en la Salud Mental. Terapia Psicológica. Vol 10. n. ${ }^{\circ}$ 2. pp 179-185. Recuperado de http://dx.doi.org/10.4067/s071848082010000200006

Palva, A. (2006). La educación liberadora de Paulo Freire y el desarrollo del pensamiento. Revista Ciencias de la Educación. Año 5, Vol 2.
No. 26, Jul-Dic 2006. Pp 133-142. Valencia. Recuperado de http:// servicio.cid.uc.edu.ve/educacion/ revista/index.htm

Organización Mundial de la Salud (1997). La salud mental de los refugiados. Biblioteca de la OMS: Ginebra Real Academia Española. (2017). Disquisición. En Diccionario de la lengua española (22. ${ }^{a}$ ed.). Recuperado de http://buscon.rae.es/drael/ SrvltConsulta?TIPO_BUS=3\&LE$M A=$ disquisici\%F3n

Watzlawick, P., Janet Beavin, Bavelas Don D. Jackson (1991).Teoría de la comunicación humana. Barcelona. Editorial Herder. 